Bull. Korean Math. Soc. 49 (2012), No. 6, pp. 1163-1178

http://dx.doi.org/10.4134/BKMS.2012.49.6.1163

\title{
EINSTEIN HALF LIGHTLIKE SUBMANIFOLDS WITH SPECIAL CONFORMALITIES
}

\author{
DAE Ho JIN
}

\begin{abstract}
In this paper, we study the geometry of Einstein half lightlike submanifolds $M$ of a semi-Riemannian space form $\bar{M}(c)$ subject to the conditions: (a) $M$ is screen conformal, and (b) the coscreen distribution of $M$ is a conformal Killing one. The main result is a classification theorem for screen conformal Einstein half lightlike submanifolds of a Lorentzian space form with a conformal Killing coscreen distribution.
\end{abstract}

\section{Introduction}

A submanifold $M$ of a semi-Riemannian manifold $(\bar{M}, \bar{g})$ is called a lightlike submanifold of $\bar{M}$ if its radical distribution $\operatorname{Rad}(T M)=T M \cap T M^{\perp}$ is a vector subbundle of the tangent bundle $T M$, of rank $r(>0)$. A codimension 2 lightlike submanifold $M$ is called a half lightlike submanifold if $\operatorname{rank}(\operatorname{Rad}(T M))=1$. Then there exists two complementary non-degenerate distributions $S(T M)$ and $S\left(T M^{\perp}\right)$ of $\operatorname{Rad}(T M)$ in $T M$ and $T M^{\perp}$ respectively, which called the screen and coscreen distribution on $M$, such that

$$
T M=\operatorname{Rad}(T M) \oplus_{\text {orth }} S(T M), T M^{\perp}=\operatorname{Rad}(T M) \oplus_{\text {orth }} S\left(T M^{\perp}\right),
$$

where the symbol $\oplus_{\text {orth }}$ denotes the orthogonal direct sum. We denote such a half lightlike submanifold by $M=(M, g, S(T M))$. Denote by $F(M)$ the algebra of smooth functions on $M$ and by $\Gamma(E)$ the $F(M)$ module of smooth sections of any vector bundle $E$ over $M$. Then there exist a non-null section $u$ on $S\left(T M^{\perp}\right)$ and a null section $\xi$ on $\operatorname{Rad}(T M)$ such that

$$
\bar{g}(u, u)=\epsilon, \quad \bar{g}(\xi, v)=0, \forall v \in \Gamma\left(T M^{\perp}\right),
$$

where $\epsilon= \pm 1$. Consider the orthogonal complementary distribution $S(T M)^{\perp}$ to $S(T M)$ in $T \bar{M}$. Certainly $\xi$ and $u$ belong to $\Gamma\left(S(T M)^{\perp}\right)$. Thus we have

$$
S(T M)^{\perp}=S\left(T M^{\perp}\right) \oplus_{\text {orth }} S\left(T M^{\perp}\right)^{\perp},
$$

Received June 18, 2009.

2010 Mathematics Subject Classification. Primary 53C25, 53C40, 53C50.

Key words and phrases. half lightlike submanifold, screen conformal, conformal Killing distribution

(C)2012 The Korean Mathematical Society 
where $S\left(T M^{\perp}\right)^{\perp}$ is the orthogonal complementary to $S\left(T M^{\perp}\right)$ in $S(T M)^{\perp}$. For any null section $\xi$ of $\operatorname{Rad}(T M)$ on a coordinate neighborhood $\mathcal{U} \subset M$, there exists a uniquely defined vector field $N \in \Gamma(\operatorname{ltr}(T M))[4]$ satisfying

$$
\bar{g}(\xi, N)=1, \bar{g}(N, N)=\bar{g}(N, X)=\bar{g}(N, u)=0, \forall X \in \Gamma(S(T M)) .
$$

We call $\operatorname{lt}(T M), N$ and $\operatorname{tr}(T M)=S\left(T M^{\perp}\right) \oplus_{\text {orth }} \operatorname{ltr}(T M)$ the lightlike transversal vector bundle, lightlike transversal vector field and transversal vector bundle of $M$ with respect to $S(T M)$ respectively. Then the tangent bundle $T \bar{M}$ of the ambient manifold $\bar{M}$ is decomposed as follows:

$$
\begin{aligned}
T \bar{M} & =T M \oplus \operatorname{tr}(T M)=\{\operatorname{Rad}(T M) \oplus \operatorname{tr}(T M)\} \oplus_{\text {orth }} S(T M) \\
& =\{\operatorname{Rad}(T M) \oplus \operatorname{ltr}(T M)\} \oplus_{\text {orth }} S\left(T M^{\perp}\right) \oplus_{\text {orth }} S(T M) .
\end{aligned}
$$

Example 1. Suppose $M$ is a surface $M$ of $R_{1}^{4}$ given by the equations

$$
x_{3}=\sqrt{x_{1}^{2}-x_{2}^{2}}, \quad x_{4}=\sqrt{1+x_{1}^{2}} .
$$

Then we derive $T M=\operatorname{Span}\{\xi, U\}$ and $T M^{\perp}=\operatorname{Span}\{\xi, u\}$, where

$$
\begin{aligned}
& U=x_{3} x_{4} \partial_{1}+x_{1} x_{4} \partial_{3}+x_{1} x_{3} \partial_{4}, \\
& \xi=x_{1} \partial_{1}+x_{2} \partial_{2}+x_{3} \partial_{3}, \quad u=x_{1} \partial_{1}+x_{4} \partial_{4} .
\end{aligned}
$$

It follows that $\operatorname{Rad}(T M)$ is a distribution on $M$ of rank 1 spanned by $\xi$. Hence $M$ is a half-lightlike submanifold of $R_{1}^{4}$ such that $S(T M)=\operatorname{Span}\{U\}$ and $S\left(T M^{\perp}\right)=\operatorname{Span}\{u\}$. Then the lightlike transversal bundle $\operatorname{ltr}(T M)$ and the transversal bundle $\operatorname{tr}(T M)$ with respect to the screen distribution $S(T M)$ are given by $\operatorname{ltr}(T M)=\operatorname{Span}\{N\}$ and $\operatorname{tr}(T M)=\operatorname{Span}\{N, u\}$, where

$$
N=-\frac{1}{2 x_{1}^{2}}\left(x_{1} \partial_{1}-x_{2} \partial_{2}-x_{3} \partial_{3}\right) \text {. }
$$

The classification of Einstein hypersurfaces $M$ in Euclidean spaces $\mathbb{R}^{n+1}$ was first studied by Fialkow [7] and Thomas [14] in the middle of 1930's. It was proved that if $M$ is a connected Einstein hypersurface $(n \geq 3)$ such that Ric $=\gamma g$ for some constant $\gamma$, then $\gamma$ is non-negative. Moreover,

(1) if $\gamma>0$, then $M$ is contained in an $n$-sphere and

(2) if $\gamma=0$, then $M$ is locally isometric to $\mathbb{R}^{n}$.

The objective of this paper is the study of half lightlike version of above classical results. For this reason, we consider only screen conformal half lightlike submanifolds with a conformal Killing coscreen distribution. In Section 2, we investigate geometric properties for screen conformal half lightlike submanifolds $M$ of a semi-Riemannian space form $\left(\bar{M}^{m+3}(c), \bar{g}\right), m>2$, with a conformal Killing coscreen distribution. In the last Section 3, we prove our main classification theorem for screen conformal Einstein half lightlike submanifolds $M$ of a Lorentzian space form with a conformal Killing coscreen distribution (Theorem 3.2 ). Recall the following structure equations. 
Let $\bar{\nabla}$ be the Levi-Civita connection of $\bar{M}$ and $P$ the projection morphism of $T M$ on $S(T M)$ with respect to the decomposition (1.1). Then the local Gauss and Weingartan formulas $M$ and $S(T M)$ are given respectively by

$$
\begin{aligned}
& \bar{\nabla}_{X} Y=\nabla_{X} Y+B(X, Y) N+D(X, Y) u, \\
& \bar{\nabla}_{X} N=-A_{N} X+\tau(X) N+\rho(X) u, \\
& \bar{\nabla}_{X} u=-A_{u} X+\phi(X) N \\
& \nabla_{X} P Y=\nabla_{X}^{*} P Y+C(X, P Y) \xi, \\
& \nabla_{X} \xi=-A_{\xi}^{*} X-\tau(X) \xi,
\end{aligned}
$$

for any $X, Y \in \Gamma(T M)$, where $\nabla$ and $\nabla^{*}$ are induced linear connections on $T M$ and $S(T M)$ respectively, the bilinear forms $B$ and $D$ on $T M$ are called the local lightlike and screen second fundamental forms of $M$ respectively, $C$ is called the local radical second fundamental form on $S(T M) . A_{N}, A_{\xi}^{*}$ and $A_{u}$ are linear operators on $\Gamma(T M)$ and $\tau, \rho$ and $\phi$ are 1-forms on $T M$.

Since $\bar{\nabla}$ is torsion-free, $\nabla$ is also torsion-free, and $B$ and $D$ are symmetric. From the facts $B(X, Y)=\bar{g}\left(\bar{\nabla}_{X} Y, \xi\right)$ and $D(X, Y)=\epsilon \bar{g}\left(\bar{\nabla}_{X} Y, u\right)$, we know that $B$ and $D$ are independent of the choice of a screen distribution and satisfy

$$
B(X, \xi)=0, \quad D(X, \xi)=-\epsilon \phi(X), \quad \forall X \in \Gamma(T M) .
$$

The induced connection $\nabla$ on $M$ is not metric and satisfies

$$
\left(\nabla_{X} g\right)(Y, Z)=B(X, Y) \eta(Z)+B(X, Z) \eta(Y)
$$

for all $X, Y, Z \in \Gamma(T M)$, where $\eta$ is a 1 -form on $T M$ such that

$$
\eta(X)=\bar{g}(X, N), \forall X \in \Gamma(T M) .
$$

But we show that $\nabla^{*}$ is metric. The above three local second fundamental forms on $T M$ and $S(T M)$ are related to their shape operators by

$$
\begin{aligned}
& B(X, Y)=g\left(A_{\xi}^{*} X, Y\right), \quad \bar{g}\left(A_{\xi}^{*} X, N\right)=0, \\
& C(X, P Y)=g\left(A_{N} X, P Y\right), \quad \bar{g}\left(A_{N} X, N\right)=0, \\
& \epsilon D(X, P Y)=g\left(A_{u} X, P Y\right), \quad \bar{g}\left(A_{u} X, N\right)=\epsilon \rho(X), \\
& \epsilon D(X, Y)=g\left(A_{u} X, Y\right)-\phi(X) \eta(Y) .
\end{aligned}
$$

From (1.12), $A_{\xi}^{*}$ is $S(T M)$-valued and self-adjoint on $\Gamma(T M)$ such that

$$
A_{\xi}^{*} \xi=0 \text {. }
$$

We denote by $\bar{R}, R$ and $R^{*}$ the curvature tensors of the Levi-Civita connection $\bar{\nabla}$ of $\bar{M}$, the induced connection $\nabla$ of $M$ and the induced connection $\nabla^{*}$ on $S(T M)$ respectively. Using the Gauss-Weingarten equations for $M$ and $S(T M)$, we obtain the Gauss-Codazzi equations for $M$ and $S(T M)$ :

$$
\begin{aligned}
\bar{g}(\bar{R}(X, Y) Z, P W)= & g(R(X, Y) Z, P W) \\
& +B(X, Z) C(Y, P W)-B(Y, Z) C(X, P W) \\
& +\epsilon\{D(X, Z) D(Y, P W)-D(Y, Z) D(X, P W)\},
\end{aligned}
$$




$$
\begin{aligned}
\bar{g}(\bar{R}(X, Y) Z, \xi)= & \left(\nabla_{X} B\right)(Y, Z)-\left(\nabla_{Y} B\right)(X, Z) \\
& +B(Y, Z) \tau(X)-B(X, Z) \tau(Y) \\
& +D(Y, Z) \phi(X)-D(X, Z) \phi(Y), \\
\bar{g}(\bar{R}(X, Y) Z, N)= & \bar{g}(R(X, Y) Z, N) \\
& +\epsilon\{D(X, Z) \rho(Y)-D(Y, Z) \rho(X)\}, \\
\bar{g}(\bar{R}(X, Y) \xi, N)= & g\left(A_{\xi}^{*} X, A_{N} Y\right)-g\left(A_{\xi}^{*} Y, A_{N} X\right) \\
& +\rho(X) \phi(Y)-\rho(Y) \phi(X)-2 d \tau(X, Y), \\
\bar{g}(\bar{R}(X, Y) Z, u)= & \epsilon\left\{\left(\nabla_{X} D\right)(Y, Z)-\left(\nabla_{Y} D\right)(X, Z)\right. \\
& +B(Y, Z) \rho(X)-B(X, Z) \rho(Y)\},
\end{aligned}
$$

$$
\begin{aligned}
\bar{g}(R(X, Y) P Z, P W)= & g\left(R^{*}(X, Y) P Z, P W\right) \\
& +C(X, P Z) B(Y, P W)-C(Y, P Z) B(X, P W), \\
g(R(X, Y) P Z, N)= & \left(\nabla_{X} C\right)(Y, P Z)-\left(\nabla_{Y} C\right)(X, P Z) \\
& +C(X, P Z) \tau(Y)-C(Y, P Z) \tau(X)
\end{aligned}
$$

for all $X, Y, Z \in \Gamma(T M)$. The Ricci curvature tensor Ric of $\bar{M}$ and the induced Ricci type tensor $R^{(0,2)}$ of $M$ are defined by

$$
\begin{aligned}
& \overline{\operatorname{Ric}}(X, Y)=\operatorname{trace}\{Z \rightarrow \bar{R}(Z, X) Y\}, \quad \forall X, Y \in \Gamma(T \bar{M}), \\
& R^{(0,2)}(X, Y)=\operatorname{trace}\{Z \rightarrow R(Z, X) Y\}, \quad \forall X, Y \in \Gamma(T M) .
\end{aligned}
$$

Consider the induced quasi-orthonormal frame fields $\left\{\xi ; W_{a}\right\}$ on $M$ such that $\operatorname{Rad} T M=\operatorname{Span}\{\xi\}$ and $S(T M)=\operatorname{Span}\left\{W_{a}\right\}$ and let $E=\left\{\xi, W_{a} ; u, N\right\}$ be the corresponding frame fields on $\bar{M}$. Let $\epsilon_{a}=g\left(W_{a}, W_{a}\right)$ be the sign of $W_{\alpha}$. Using this quasi-orthonormal frame, (1.24) and (1.25) reduce respectively to

$$
\begin{aligned}
& \overline{\operatorname{Ric}}(X, Y)=\sum_{a=1}^{m} \epsilon_{a} \bar{g}\left(\bar{R}\left(W_{a}, X\right) Y, W_{a}\right)+\bar{g}(\bar{R}(\xi, X) Y, N) \\
& +\epsilon \bar{g}(\bar{R}(u, X) Y, u)+\bar{g}(\bar{R}(N, X) Y, \xi), \\
& R^{(0,2)}(X, Y)=\sum_{a=1}^{m} \epsilon_{a} g\left(R\left(W_{a}, X\right) Y, W_{a}\right)+\bar{g}(R(\xi, X) Y, N)
\end{aligned}
$$

for any $X, Y \in \Gamma(T M)$. Substituting (1.17) and (1.19) in (1.26) and then, using (1.12), (1.13) and (1.27), we obtain

$$
\begin{aligned}
R^{(0,2)}(X, Y)= & \overline{\operatorname{Ric}}(X, Y)+B(X, Y) \operatorname{tr} A_{N}+D(X, Y) \operatorname{tr} A_{u} \\
& -g\left(A_{N} X, A_{\xi}^{*} Y\right)-\epsilon g\left(A_{u} X, A_{u} Y\right)+\rho(X) \phi(Y) \\
& -\bar{g}(\bar{R}(\xi, Y) X, N)-\epsilon \bar{g}(\bar{R}(u, Y) X, u)
\end{aligned}
$$

for any $X, Y \in \Gamma(T M)$. A tensor field $R^{(0,2)}$ of $M$ is called its induced Ricci tensor if it is symmetric. A symmetric $R^{(0,2)}$ tensor will be denoted by Ric. 
Note 1. Using (1.20), (1.28) and the first Bianchi's identity, we obtain

$$
R^{(0,2)}(X, Y)-R^{(0,2)}(Y, X)=2 d \tau(X, Y), \quad \forall X, Y \in \Gamma(T M) .
$$

It follow that $R^{(0,2)}$ is a symmetric, if and only if, each 1-form $\tau$ is closed, i.e., $d \tau=0$, on any $\mathcal{U} \subset M[6]$. Therefore, suppose $R^{(0,2)}$ is symmetric, then there exists a smooth function $f$ on $\mathcal{U}$ such that $\tau=d f$. Consequently we get $\tau(X)=X(f)$. If we take $\bar{\xi}=\alpha \xi$, it follows that $\tau(X)=\bar{\tau}(X)+X(\ln \alpha)$. Setting $\alpha=\exp (f)$ in this equation, we get $\bar{\tau}(X)=0$ for any $X \in \Gamma\left(T M_{\mid \mathcal{U}}\right)$. In the sequel, we call the pair $\{\xi, N\}$ on $\mathcal{U}$ such that the corresponding 1-form $\tau$ vanishes the canonical null pair [9] of $M$.

\section{Screen conformal submanifolds}

Definition. A half lightlike submanifold $(M, g, S(T M))$ of $\bar{M}$ is said to be screen conformal [1] if there exists a non-vanishing smooth function $\varphi$ on a neighborhood $\mathcal{U}$ in $M$ such that $A_{N}=\varphi A_{\xi}^{*}$, or equivalently,

$$
C(X, P Y)=\varphi B(X, Y), \forall X, Y \in \Gamma(T M) .
$$

In general, $S(T M)$ is not necessarily integrable. From (1.7) and (1.13), we get $g\left(A_{N} X, Y\right)-g\left(X, A_{N} Y\right)=C(X, Y)-C(Y, X)=\eta([X, Y])$ for all $X, Y \in \Gamma(S(T M))$. Thus $A_{N}$ is self-adjoint on $S(T M)$ with respect to $g$ if and only if $C$ is symmetric on $S(T M)$ if and only if $\eta([X, Y])=0$ for all $X, Y \in \Gamma(S(T M))$, i.e., $S(T M)$ is integrable [4].

Note 2. For a screen conformal $M$, since $C$ is symmetric on $S(T M)$, the screen distribution $S(T M)$ is integrable. Thus $M$ is locally a product manifold $L \times M^{*}$ where $L$ is a null curve and $M^{*}$ is a leaf of $S(T M)$ [5].

Example 2. Consider a surface $M$ in $R_{2}^{5}$ given by the equation

$$
x_{4}=\sqrt{x_{1}^{2}+x_{2}^{2}}, \quad x_{5}=\sqrt{1-x_{3}^{2}} .
$$

Then we have $T M=\operatorname{Span}\{\xi, U, V\}$ and $T M^{\perp}=\operatorname{Span}\{\xi, u\}$, where

$$
\begin{array}{ll}
U=x_{4} \partial_{1}+x_{1} \partial_{4}, & V=x_{5} \partial_{3}-x_{3} \partial_{5}, \\
\xi=x_{1} \partial_{1}+x_{2} \partial_{2}+x_{4} \partial_{4}, & u=x_{3} \partial_{3}+x_{5} \partial_{5} .
\end{array}
$$

By direct calculations we check that $\operatorname{Rad}(T M)$ is a distribution on $M$ of rank 1 spanned by $\xi$. Hence $M$ is a half-lightlike submanifold of $R_{2}^{5}$ such that $S(T M)=\operatorname{Span}\{U, V\}$ and $S\left(T M^{\perp}\right)=\operatorname{Span}\{u\}$. Then the lightlike transversal bundle $\operatorname{lt}(T M)$ of the screen $S(T M)$ is given by

$$
\operatorname{ltr}(T M)=\operatorname{Span}\left\{N=\frac{1}{2 x_{2}^{2}}\left(x_{1} \partial_{1}-x_{2} \partial_{2}+x_{4} \partial_{4}\right)\right\}
$$

and the transversal bundle $\operatorname{tr}(T M)$ is given by $\operatorname{tr}(T M)=\operatorname{Span}\{N, u\}$. 
Denote by $\bar{\nabla}$ the Levi-Civita connection on $R_{2}^{5}$. By straightforward calculations, we obtain

$$
\begin{aligned}
& \bar{\nabla}_{U} U=\xi+2 x_{2}^{2} N, \quad \bar{\nabla}_{U} V=0, \quad \bar{\nabla}_{U} \xi=2 U+\frac{x_{1} x_{4}}{2 x_{2}^{2}} \xi-x_{1} x_{4} N, \\
& \bar{\nabla}_{U} N=\frac{1}{2 x_{2}^{2}} U-2 \frac{x_{1} x_{4}}{x_{2}^{2}} N+\frac{x_{1} x_{4}}{x_{2}^{2}} \xi, \quad \bar{\nabla}_{U} u=0, \\
& \bar{\nabla}_{V} U=0, \quad \bar{\nabla}_{V} V=-2 u, \quad \bar{\nabla}_{V} \xi=0, \quad \bar{\nabla}_{V} N=0, \quad \bar{\nabla}_{V} u=2 V, \\
& \bar{\nabla}_{\xi} U=U+\frac{x_{4}}{2 x_{1}} \xi+\frac{x_{2}^{2} x_{4}}{x_{1}} N, \quad \bar{\nabla}_{\xi} \xi=\frac{x_{4}}{x_{1}} U+\left(\frac{3}{2}+\frac{x_{1}^{2}}{2 x_{2}^{2}}\right) \xi-x_{4}^{2} N, \\
& \bar{\nabla}_{\xi} V=0, \quad \bar{\nabla}_{\xi} N=-N, \quad \bar{\nabla}_{\xi} u=0 .
\end{aligned}
$$

Then taking into account of Gauss and Weingarten formulas infer

$$
\begin{gathered}
A_{\xi}^{*} U=-U, \quad A_{\xi}^{*} V=0, \quad A_{N} U=-\frac{1}{2 x_{4}^{2}} U, \quad A_{N} V=0, \quad A_{N} \xi=0, \\
\tau(U)=\tau(V)=\tau(\xi)=0, \quad \rho(U)=\rho(V)=\rho(\xi)=0 .
\end{gathered}
$$

Thus $A_{N} X=\left(1 / 2 x_{4}^{2}\right) A_{\xi}^{*} X$ for any $X \in \Gamma(T M)$ and $M$ is a screen conformal half-lightlike submanifold of $R_{2}^{5}$ with a conformal factor $\varphi=1 / 2 x_{2}^{2}$.

Definition. A vector field $X$ on $\bar{M}$ is said to be a conformal Killing [15] if $\overline{\mathcal{L}}_{X} \bar{g}=-2 \delta \bar{g}$, where $\delta$ is a non-vanishing smooth function on $\bar{M}$ and $\overline{\mathcal{L}}_{X}$ denotes the Lie derivative with respect to $X$. In particular, if $\delta=0$, then $X$ is called a Killing. A distribution $\mathcal{G}$ on $\bar{M}$ is said to be a conformal Killing (Killing) if each vector field belonging to $\mathcal{G}$ is a conformal Killing (Killing).

Theorem 2.1. Let $(M, g, S(T M))$ be a half lightlike submanifold of a semiRiemannian manifold $(\bar{M}, \bar{g})$. Then the coscreen distribution is a conformal Killing if and only if $D(X, Y)=\epsilon \delta g(X, Y)$ for any $X, Y \in \Gamma(T M)$.

Proof. By straightforward calculations and use (1.6) and (1.15), we have

$$
\begin{gathered}
\left(\overline{\mathcal{L}}_{u} \bar{g}\right)(X, Y)=\bar{g}\left(\bar{\nabla}_{X} u, Y\right)+\bar{g}\left(X, \bar{\nabla}_{Y} u\right), \\
\bar{g}\left(\bar{\nabla}_{X} u, Y\right)=-g\left(A_{u} X, Y\right)+\phi(X) \eta(Y)=-\epsilon D(X, Y)
\end{gathered}
$$

for any $X, Y \in \Gamma(T M)$. Therefore, we obtain $\left(\overline{\mathcal{L}}_{u} \bar{g}\right)(X, Y)=-2 \epsilon D(X, Y)$.

Let $(M, g, S(T M))$ be a screen conformal half lightlike submanifold of a semi-Riemannian space form $(\bar{M}(c), \bar{g})$ with a conformal Killing coscreen. For all $X, Y, Z, W \in \Gamma(T M)$, by (1.9), (1.14) and (1.15), we have

$$
D(X, Y)=\epsilon \delta g(X, Y), \quad \phi(X)=0, \quad A_{u} X=\delta P X+\epsilon \rho(X) \xi .
$$

Using (2.1) and (2.2), the Gauss equations (1.17) and (1.22) reduce to

$$
\begin{aligned}
& g(R(X, Y) Z, P W) \\
= & \left(c+\epsilon \delta^{2}\right)\{g(Y, Z) g(X, P W)-g(X, Z) g(Y, P W)\} \\
& +\varphi\{B(Y, Z) B(X, P W)-B(X, Z) B(Y, P W)\},
\end{aligned}
$$




$$
\begin{aligned}
& g\left(R^{*}(X, Y) P Z, P W\right) \\
= & \left(c+\epsilon \delta^{2}\right)\{g(Y, P Z) g(X, P W)-g(X, P Z) g(Y, P W)\} \\
& +2 \varphi\{B(Y, P Z) B(X, P W)-B(X, P Z) B(Y, P W)\}
\end{aligned}
$$

respectively. From (1.18) with $\phi=0$ and (1.21), we have

$$
\begin{aligned}
& \left(\nabla_{X} B\right)(Y, Z)-\left(\nabla_{Y} B\right)(X, Z)=B(X, Z) \tau(Y)-B(Y, Z) \tau(X), \\
& \left(\nabla_{X} D\right)(Y, Z)-\left(\nabla_{Y} D\right)(X, Z)=B(X, Z) \rho(Y)-B(Y, Z) \rho(X) .
\end{aligned}
$$

Differentiating the first equation of $(2.2)$ and using (2.6), we have

$$
\begin{aligned}
& \{\delta \eta(X)-\epsilon \rho(X)\} B(Y, Z)-\{\delta \eta(Y)-\epsilon \rho(Y)\} B(X, Z) \\
= & X[\delta] g(Y, Z)-Y[\delta] g(X, Z) .
\end{aligned}
$$

Replacing $Y$ by $\xi$ in the last equation and using (1.9), we obtain

$$
\{\delta-\epsilon \rho(\xi)\} B(X, Z)=\xi[\delta] g(X, Z) .
$$

Using (1.19), (1.23), (2.1) and (2.5), we obtain

$$
\begin{aligned}
& \{X[\varphi]-2 \varphi \tau(X)\} B(Y, P Z)-\{Y[\varphi]-2 \varphi \tau(Y)\} B(X, P Z) \\
= & \{c \eta(X)+\delta \rho(X)\} g(Y, P Z)-\{c \eta(Y)+\delta \rho(Y)\} g(X, P Z) .
\end{aligned}
$$

Replacing $Y$ by $\xi$ in the last equation and using (1.9), we obtain

$$
\{\xi[\varphi]-2 \varphi \tau(\xi)\} B(X, P Z)=(c+\delta \rho(\xi)) g(X, P Z) .
$$

Theorem 2.2. Let $(M, g, S(T M))$ be a screen conformal half lightlike submanifold of a semi-Riemannian space form $\left(\bar{M}^{m+3}(c), \bar{g}\right), m>2$, with a conformal Killing coscreen distribution. Then we have $c+\delta \rho(\xi)=0$.

Proof. Assume that $c+\delta \rho(\xi) \neq 0$. Then we have $\xi[\varphi]-2 \varphi \tau(\xi) \neq 0$ and $B \neq 0$ by virtue of (2.10). Thus, from (1.9), (2.1) and (2.10), we have

(2.11) $B(X, Y)=\sigma g(X, Y), C(X, P Y)=\varphi \sigma g(X, Y), \forall X, Y \in \Gamma(T M)$, where $\sigma=(c+\delta \rho(\xi))(\xi[\varphi]-2 \varphi \tau(\xi))^{-1} \neq 0$. From the first equation of (2.2) and (2.11), $M$ is totally umbilical in $\bar{M}$ and $S(T M)$ is also totally umbilical in $M$ and $\bar{M}$. As $\bar{M}$ has a constant curvature $c$, from (2.4) and (2.11), we have

$$
R^{*}(X, Y) Z=\left(c+2 \varphi \sigma^{2}+\epsilon \delta^{2}\right)\{g(Y, Z) X-g(X, Z) Y\}
$$

for all $X, Y, Z \in \Gamma(S(T M))$. Let $M^{*}$ be the leaf of $S(T M)$ and $R i c^{*}$ be the Ricci tensor of $M^{*}$. Then, from the last equation, we have

$$
\operatorname{Ric}^{*}(X, Y)=\left(c+2 \varphi \sigma^{2}+\epsilon \delta^{2}\right)(m-1) g(X, Y), \quad \forall X, Y \in \Gamma(S(T M)) .
$$

Thus $M^{*}$ is Einstein. As $m>2,\left(c+2 \varphi \sigma^{2}+\epsilon \delta^{2}\right)$ is a constant and $M^{*}$ is a space of constant curvature $\left(c+2 \varphi \sigma^{2}+\epsilon \delta^{2}\right)$. Differentiating the first equation of (2.11) and using (1.10) and (2.5), we have

$$
\left\{X[\sigma]+\sigma \tau(X)-\sigma^{2} \eta(X)\right\} g(Y, Z)=\left\{Y[\sigma]+\sigma \tau(Y)-\sigma^{2} \eta(Y)\right\} g(X, Z)
$$

for all $X, Y, Z \in \Gamma(T M)$. Replacing $Y$ by $\xi$ in this equation, we have $\xi[\sigma]=$ $\sigma^{2}-\sigma \tau(\xi)$. From (2.8) and (2.11), we have $\xi[\delta]=\sigma \delta-\epsilon \sigma \rho(\xi)$. Since $\left(c+2 \varphi \sigma^{2}+\right.$ 
$\left.\epsilon \delta^{2}\right)$ is a constant, we have $\xi\left[c+2 \varphi \sigma^{2}+\epsilon \delta^{2}\right]=2 \sigma\left(c+2 \varphi \sigma^{2}+\epsilon \delta^{2}\right)=0$. Therefore, as $\sigma \neq 0$, we have $c+2 \varphi \sigma^{2}+\epsilon \delta^{2}=0$ and consequently we get $R^{*}=0$. Thus $M^{*}$ is a semi-Euclidean space. As the second fundamental form of the totally umbilical semi-Euclidean space $M^{*}$ as a submanifold of the semi-Riemannian space form $\bar{M}(c)$ vanishes [3, Section 2.3], we get $C=0$. Consequently, from (2.1), we get $B=0$ and $c+\delta \rho(\xi)=0$ due to (2.10). It is a contradiction to $c+\delta \rho(\xi) \neq 0$. Thus we have $c+\delta \rho(\xi)=0$.

Corollary $2.3([10])$. Let $(M, g, S(T M))$ be a screen conformal half lightlike submanifold of a semi-Riemannian space form $\left(\bar{M}^{m+3}(c), \bar{g}\right), m>2$, with a Killing coscreen distribution. Then we have $c=0$ and $\delta=0$.

Theorem 2.4. Let $(M, g, S(T M))$ be a screen conformal Einstein half lightlike submanifold of a semi-Riemannian space form $\left(\bar{M}^{m+3}(c), \bar{g}\right), m>2$, with a conformal Killing coscreen distribution of conformal factor $\delta$. Then the leaf $M^{*}$ of $S(T M)$ is an Einstein manifold and $\delta$ is a constant.

Proof. From (2.3) and (2.4), we show that

$$
\begin{aligned}
& 2 g(R(X, Y) P Z, P W) \\
= & g\left(R^{*}(X, Y) P Z, P W\right)+\left(c+\epsilon \delta^{2}\right)\{g(Y, P Z) g(X, P W)-g(X, P Z) g(Y, P W)\}
\end{aligned}
$$

for all $X, Y, Z, W \in \Gamma(T M)$. Using the equations (1.27), (2.12) and the fact that $\bar{g}(R(\xi, X) Y, N)=(c+\delta \rho(\xi)) g(X, Y)=0$, we get

$$
2 R^{(0,2)}(X, Y)=\operatorname{Ric}^{*}(X, Y)+(m-1)\left(c+\epsilon \delta^{2}\right) g(X, Y) .
$$

This shows that the induced tensor $R^{(0,2)}$ on $M$ is symmetric. Thus $M$ admits a symmetric Ricci tensor and $R^{(0,2)}=$ Ric. Since $M$ is Einstein, i.e., Ric $=\gamma g$, where $\gamma$ is a constant if $m>2$, the last equation reduces to

$$
\operatorname{Ric}^{*}(X, Y)=\left\{2 \gamma-(m-1)\left(c+\epsilon \delta^{2}\right)\right\} g(X, Y), \forall X, Y \in \Gamma(T M) .
$$

Thus $M^{*}$ is also Einstein. Since $m>2$, the function $\left\{2 \gamma-(m-1)\left(c+\epsilon \delta^{2}\right)\right\}$ is a constant. Therefore, the conformal factor $\delta$ is a constant.

Theorem 2.5. Let $(M, g, S(T M))$ be a screen conformal Einstein half lightlike submanifold of a semi-Riemannian space form $\left(\bar{M}^{m+3}(c), \bar{g}\right), m>2$, with a conformal Killing coscreen distribution of conformal factor $\delta$. If either $\gamma \neq$ $(m-1)\left(c+\epsilon \delta^{2}\right)$ or $\operatorname{rank} A_{\xi}^{*}>0$, then we have $c+\epsilon \delta^{2}=0$.

Proof. Since $M$ is Einstein, the conformal factor $\delta$ is a constant by Theorem 2.4. From (2.8) with $c+\delta \rho(\xi)=0$, we get $\left\{c+\epsilon \delta^{2}\right\} B(Y, Z)=0$, or equivalently, $\left\{c+\epsilon \delta^{2}\right\} A_{\xi}^{*} X=0$ for any $X, Y \in \Gamma(T M)$. First, if rank $A_{\xi}^{*}>0$, we get $c+\epsilon \delta^{2}=0$. Next, if $c+\epsilon \delta^{2} \neq 0$, then, since $\left(c+\epsilon \delta^{2}\right)$ is a constant, we have $B(X, Y)=0$ for any $X, Y \in \Gamma(T M)$. Thus, from $(1.27),(2.3)$ and the fact that $\bar{g}(R(\xi, X) Y, N)=(c+\delta \rho(\xi)) g(X, Y)=0$, we have $\gamma=(m-1)\left(c+\epsilon \delta^{2}\right)$. This implies that if $\gamma \neq(m-1)\left(c+\epsilon \delta^{2}\right)$, then we get $c+\epsilon \delta^{2}=0$. 
Recall the following notion of null sectional curvature $[2,5,6,8]$. Let $x \in M$ and $\xi$ be a null vector of $T_{x} M$. A plane $H$ of $T_{x} M$ is called a null plane directed by $\xi$ if it contains $\xi, g_{x}(\xi, W)=0$ for any $W \in H$ and there exists $W_{o} \in H$ such that $g_{x}\left(W_{o}, W_{o}\right) \neq 0$. Then, the null sectional curvature of $H$, with respect to $\xi$ and $\nabla$, is defined as a real number

$$
K_{\xi}(H)=\frac{g_{x}(R(\xi, W) W, \xi)}{g_{x}(W, W)},
$$

where $W \neq 0$ is any vector in $H$ independent with $\xi$. It is easy to see that $K_{\xi}(H)$ is independent of $W$ but depends in a quadratic fashion on $\xi$. An $n(\geq 3)$-dimensional Lorentzian manifold is of constant curvature if and only if its null sectional curvatures are everywhere zero [12].

Theorem 2.6. Let $(M, g, S(T M))$ be a screen conformal half lightlike submanifold of a semi-Riemannian space form $\left(\bar{M}^{m+3}(c), \bar{g}\right), m>2$, with a conformal Killing coscreen distribution. Then every null plane $H$ of $T_{x} M$ directed by $\xi$ has everywhere zero null sectional curvatures.

Proof. From (1.9), (1.19) and (2.3), we show that $g(R(\xi, X) Y, P W)=0$ and $g(R(\xi, X) Y, N)=(c+\delta \rho(\xi)) g(X, Y)=0$ for any $X, Y \in \Gamma(T M)$. Thus the curvature tensor $R$ of $M$ satisfies $R(\xi, X) Y=0$ for any $X, Y \in \Gamma(T M)$. Thus $K_{\xi}(H)=\frac{g_{x}(R(\xi, W) W, \xi)}{g_{x}(W, W)}=0$ for any null plane $H$ of $T_{x} M$ directed by $\xi$.

\section{Einstein submanifolds}

In this section, let $(M, g, S(T M))$ be a screen conformal half lightlike submanifold of a Lorentzian space form $(\bar{M}(c), \bar{g})$ with a conformal Killing coscreen distribution. Then $\epsilon=1, \phi=0$ and $S(T M)$ is a Riemannian and integrable vector bundle. As $\bar{M}$ is a Lorentzian space form, then $\bar{R}(\xi, Y) X=c \bar{g}(X, Y) \xi$, $\bar{R}(u, X) Y=c \bar{g}(X, Y) u$ and $\overline{\operatorname{Ri}} c(X, Y)=(m+2) c \bar{g}(X, Y)$. Thus the equation (1.28) reduces to

$$
\begin{aligned}
\operatorname{Ric}(X, Y)= & m c g(X, Y)+B(X, Y) \operatorname{tr} A_{N}+D(X, Y) \operatorname{tr} A_{u} \\
& -\varphi g\left(A_{\xi}^{*} X, A_{\xi}^{*} Y\right)-g\left(A_{u} X, A_{u} Y\right), \quad \forall X, Y \in \Gamma(T M) .
\end{aligned}
$$

From (1.16), $\xi$ is an eigenvector field of $A_{\xi}^{*}$ corresponding to the eigenvalue 0 . Since $A_{\xi}^{*}$ is $\Gamma(S(T M))$-valued real self-adjoint operator on $\Gamma(T M)$ with respect to $g, A_{\xi}^{*}$ have $m$ real orthonormal eigenvector fields in $S(T M)$ and is diagonalizable. Consider a frame field of eigenvectors $\left\{\xi, E_{1}, \ldots, E_{m}\right\}$ of $A_{\xi}^{*}$ such that $\left\{E_{1}, \ldots, E_{m}\right\}$ is an orthonormal frame field of $S(T M)$. Then

$$
A_{\xi}^{*} E_{i}=\lambda_{i} E_{i}, \quad 1 \leq i \leq m .
$$

Let $M$ be an Einstein manifold. Then Ric $=\gamma g$ and (3.1) reduces to

$$
g\left(A_{\xi}^{*} X, A_{\xi}^{*} Y\right)-s g\left(A_{\xi}^{*} X, Y\right)+F g(X, Y)=0,
$$


where $s=\operatorname{tr} A_{\xi}^{*}$ is the trace of $A_{\xi}^{*}$ and $F=\varphi^{-1}\left\{\gamma-m c-\delta \rho(\xi)+(1-m) \delta^{2}\right\}$ is a smooth function. In case $m>2$, we show that $F=\varphi^{-1}\left\{\gamma-(m-1)\left(c+\delta^{2}\right)\right\}$. Put $X=Y=E_{i}$ in (3.2), the eigenvalue $\lambda_{i}$ is a solution of

$$
x^{2}-s x+F=0 .
$$

The equation (3.3) has at most two distinct solutions. Assume that there exists $p \in\{0,1, \ldots, m\}$ such that $\lambda_{1}=\cdots=\lambda_{p}=\alpha$ and $\lambda_{p+1}=\cdots=\lambda_{m}=\beta$, by renumbering if necessary. From (3.3), we have

$$
s=\alpha+\beta=p \alpha+(m-p) \beta, \quad \alpha \beta=F .
$$

Although $S(T M)$ is not unique, it is canonically isomorphic to the factor vector bundle $S(T M)^{\sharp}=T M / \operatorname{Rad} T M$ considered by Kupeli [11]. Thus all $S(T M)$ are isomorphic. For this reason, in the sequel, let $(M, g, S(T M))$ be a screen conformal Einstein half lightlike submanifold equipped with the canonical null pair $\{\xi, N\}$ of a Lorentzian space form $\left(\bar{M}^{m+3}(c), \bar{g}\right), m>2$, with a conformal Killing coscreen distribution.

Theorem 3.1. Let $(M, g, S(T M))$ be a screen conformal Einstein half lightlike submanifold of a Lorentzian space form $\left(\bar{M}^{m+3}(c), \bar{g}\right), m>2$, with a conformal Killing coscreen distribution. Then $M$ is locally a product manifold $L \times M_{\alpha} \times$ $M_{\beta}$, where $L$ is a null curve and $M_{\alpha}$ and $M_{\beta}$ are totally umbilical leaves of some distributions of $M$.

Proof. If (3.3) has only one solution $\alpha$, then, since $M$ is screen conformal, $M=L \times M^{*} \cong L \times M^{*} \times\{x\}$ for any $x \in \bar{M}$, where $M_{\alpha}=M^{*}$ is a leaf of $S(T M)$ and $M_{\beta}=\{x\}$ is a leaf of the trivial vector bundle $\{0\}$. Since $B(X, Y)=g\left(A_{\xi}^{*} X, Y\right)=\alpha g(X, Y)$ for all $X, Y \in \Gamma(T M)$, we get $C(X, Y)=$ $\varphi \alpha g(X, Y)$ for all $X, Y \in \Gamma(T M)$ by (2.1). Thus $M^{*}$ is totally umbilical and $\{x\}$ is also totally umbilical. In this case, our assertion is true.

Assume that (3.3) has exactly two distinct solutions $\alpha$ and $\beta$. If $p=0$ or $p=m$, then we also show that $M=L \times M^{*} \cong L \times M^{*} \times\{x\}$ for any $x \in \bar{M}$, and $M^{*}=M_{\alpha}$ and $M_{\beta}=\{x\}$ (if $p=m$ ) or $M_{\beta}$ and $M_{\alpha}=\{x\}$ (if $p=0$ ). In these cases, $M^{*}$ is totally umbilical. If $0<p<m$. Consider the following four distributions $D_{\alpha}, D_{\beta}, D_{\alpha}^{s}$ and $D_{\beta}^{s}$ on $M$ :

$$
\begin{array}{ll}
\Gamma\left(D_{\alpha}\right)=\left\{X \in \Gamma(T M) \mid A_{\xi}^{*} X=\alpha P X\right\}, & D_{\alpha}^{s}=P D_{\alpha} ; \\
\Gamma\left(D_{\beta}\right)=\left\{U \in \Gamma(T M) \mid A_{\xi}^{*} U=\beta P U\right\}, & D_{\beta}^{s}=P D_{\beta} .
\end{array}
$$

Then $D_{\alpha} \cap D_{\beta}=\operatorname{Rad}(T M)$ and $D_{\alpha}^{s} \cap D_{\beta}^{s}=\{0\}$.

Since $A_{\xi}^{*} P X=A_{\xi}^{*} X=\alpha P X$ for all $X \in \Gamma\left(D_{\alpha}\right)$ and $A_{\xi}^{*} P U=A_{\xi}^{*} U=\beta P U$ for all $U \in \Gamma\left(D_{\beta}\right), P X$ and $P U$ are eigenvector fields of the real symmetric operator $A_{\xi}^{*}$ corresponding to the different eigenvalues $\alpha$ and $\beta$ respectively. Thus $P X \perp_{g} P U$ and $g(X, U)=g(P X, P U)=0$, that is, $D_{\alpha} \perp_{g} D_{\beta}$. Also, since $B(X, U)=g\left(A_{\xi}^{*} X, U\right)=\alpha g(P X, P U)=0$, we show that $D_{\alpha}^{g} \perp_{B} D_{\beta}$. 
For any $x \in M$, since $\left\{E_{i}\right\}_{1 \leq i \leq p}$ and $\left\{E_{a}\right\}_{p+1 \leq a \leq m}$ are $p$ and $(m-p)$ smooth linearly independent vector fields of $D_{\alpha}^{s}$ and $D_{\beta}^{s}$ respectively, $D_{\alpha}^{s}$ and $D_{\beta}^{s}$ are smooth distributions. Also, as $\left\{\xi, E_{i}\right\}_{1 \leq i \leq p}$ and $\left\{\xi, E_{a}\right\}_{p+1 \leq a \leq m}$ are $(p+1)$ and $(m-p+1)$ smooth linearly independent vector fields of $D_{\alpha}$ and $D_{\beta}$ respectively, $D_{\alpha}$ and $D_{\beta}$ are also smooth distributions on $M$. Thus $D_{\alpha}^{s}$ and $D_{\beta}^{s}$ are orthogonal vector subbundle of $S(T M), D_{\alpha}^{s}$ and $D_{\beta}^{s}$ are non-degenerate distributions of rank $p$ and rank $(m-p)$ respectively. Thus $S(T M)=D_{\alpha}^{s} \oplus_{\text {orth }}$ $D_{\beta}^{s}$. Consequently, $T M=\operatorname{Rad}(T M) \oplus_{\text {orth }} D_{\alpha}^{s} \oplus_{\text {orth }} D_{\beta}^{s}$.

From $(3.2)$, we show that $\left(A_{\xi}^{*}\right)^{2}-(\alpha+\beta) A_{\xi}^{*}+\alpha \beta P=0$. Let $Y \in \operatorname{Im}\left(A_{\xi}^{*}-\right.$ $\alpha P)$, then there exists $X \in \Gamma(T M)$ such that $Y=\left(A_{\xi}^{*}-\alpha P\right) X$. Then $\left(A_{\xi}^{*}-\right.$ $\beta P) Y=0$ and $Y \in \Gamma\left(D_{\beta}\right)$. Thus $\operatorname{Im}\left(A_{\xi}^{*}-\alpha P\right) \subset \Gamma\left(D_{\beta}\right)$. Since the morphism $A_{\xi}^{*}-\alpha P$ maps $\Gamma(T M)$ onto $\Gamma(S(T M))$, we have $\operatorname{Im}\left(A_{\xi}^{*}-\alpha P\right) \subset \Gamma\left(D_{\beta}^{s}\right)$. By duality, we also have $\operatorname{Im}\left(A_{\xi}^{*}-\beta P\right) \subset \Gamma\left(D_{\alpha}^{s}\right)$.

For $X, Y \in \Gamma\left(D_{\alpha}\right)$ and $U \in \Gamma\left(D_{\beta}\right)$, we have

$$
\left(\nabla_{X} B\right)(Y, U)=-g\left(\left(A_{\xi}^{*}-\alpha P\right) \nabla_{X} Y, U\right)+\alpha^{2} g(X, Y) \eta(U)
$$

and $\left(\nabla_{X} B\right)(Y, U)=\left(\nabla_{Y} B\right)(X, U)$ due to $(2.5)$. Thus $g\left(\left(A_{\xi}^{*}-\alpha P\right)[X, Y], U\right)=$ 0 . As $D_{\beta}^{s}$ is non-degenerate and $\operatorname{Im}\left(A_{\xi}^{*}-\alpha P\right) \subset \Gamma\left(D_{\beta}^{s}\right)$, we have $\left(A_{\xi}^{*}-\right.$ $\alpha P)[X, Y]=0$. Thus $[X, Y] \in \Gamma\left(D_{\alpha}\right)$ and $D_{\alpha}$ is integrable. By duality, $D_{\beta}$ is also integrable. Since $S(T M)$ is integrable, for any $X, Y \in \Gamma\left(D_{\alpha}^{s}\right)$, we have $[X, Y] \in \Gamma\left(D_{\alpha}\right)$ and $[X, Y] \in \Gamma(S(T M))$. Thus $[X, Y] \in \Gamma\left(D_{\alpha}^{s}\right)$ and $D_{\alpha}^{s}$ is integrable. So is $D_{\beta}^{s}$.

For $X, Y \in \Gamma\left(D_{\alpha}\right)$ and $Z \in \Gamma(T M)$, we show that

$$
\begin{aligned}
\left(\nabla_{X} B\right)(Y, Z)= & -g\left(\left(A_{\xi}^{*}-\alpha P\right) \nabla_{X} Y, Z\right)+\alpha^{2} g(X, Y) \eta(Z) \\
& +(X \alpha) g(Y, Z)+\alpha^{2} \eta(Y) g(X, Z) .
\end{aligned}
$$

Using this equation and the facts that $\left(\nabla_{X} B\right)(Y, Z)=\left(\nabla_{Y} B\right)(X, Z)$ due to (2.5) and $\left(A_{\xi}^{*}-\alpha P\right)[X, Y]=0$ for any $X, Y \in \Gamma\left(D_{\alpha}\right)$, we have

$$
\left\{X \alpha-\alpha^{2} \eta(X)\right\} g(Y, Z)=\left\{Y \alpha-\alpha^{2} \eta(Y)\right\} g(X, Z), \forall X, Y \in \Gamma\left(D_{\alpha}\right) .
$$

Therefore, for $X, Y \in \Gamma\left(D_{\alpha}^{s}\right)$ and $Z \in \Gamma(S(T M))$, we obtain $(X \alpha) g(Y, Z)=$ $(Y \alpha) g(X, Z)$. Since $S(T M)$ is non-degenerate, we have $d \alpha(X) Y=d \alpha(Y) X$. Suppose there exists a vector field $X_{o} \in \Gamma\left(D_{\alpha}^{s}\right)$ such that $d \alpha\left(X_{o}\right)_{x} \neq 0$ at each point $x \in M$, then $Y=f X_{o}$ for any $Y \in \Gamma\left(D_{\alpha}^{s}\right)$, where $f$ is a smooth function. It follows that all vectors from the fiber $\left(D_{\alpha}^{s}\right)_{x}$ are colinear with $\left(X_{o}\right)_{x}$. It is a contradiction as $\operatorname{dim}\left(\left(D_{\alpha}^{s}\right)_{x}\right)=p>1$. Thus we have $\left.d \alpha\right|_{D_{\alpha}^{s}}=0$. By duality, we also have $\left.d \beta\right|_{D_{\beta}^{s}}=0$. Thus $\alpha$ is a constant along $D_{\alpha}^{s}$ and $\beta$ is a constant along $D_{\beta}^{s}$. From the first equation of (3.4), we have $(p-1) \alpha=-(m-p-1) \beta$. Thus both $\alpha$ and $\beta$ are constants along $S(T M)$.

Using (2.9) with $c+\delta \rho(\xi)=0$ and $\tau=0$, we have

$$
(X \varphi) B(Y, Z)-(Y \varphi) B(X, Z)=\delta\{\rho(P X) g(Y, Z)-\rho(P Y) g(X, Z)\}
$$


for any $X, Y, Z \in \Gamma(T M)$. Take $X, Y, Z \in \Gamma\left(D_{\alpha}^{s}\right)$, then (3.5) reduces to

$$
\{\alpha(X \varphi)-\delta \rho(X)\} Y=\{\alpha(Y \varphi)-\delta \rho(Y)\} X .
$$

Since $\operatorname{dim}\left(D_{\alpha}^{s}\right)_{x}>1$, we have $(X \varphi) \alpha=\delta \rho(X)$ for all $X \in \Gamma\left(D_{\alpha}^{s}\right)$. While, take $X \in \Gamma\left(D_{\beta}^{s}\right)$ and $Y, Z \in \Gamma\left(D_{\alpha}^{s}\right)$ in (3.5), we have $(X \varphi) \alpha=\delta \rho(X)$ for all $X \in \Gamma\left(D_{\beta}^{s}\right)$. Consequently, we obtain $(X \varphi) \alpha=\delta \rho(X)$ for all $X \in \Gamma(S(T M))$. By duality, we get $(X \varphi) \beta=\delta \rho(X)$ for all $X \in \Gamma(S(T M))$. Thus we have $(X \varphi) \alpha=(X \varphi) \beta$ for all $X \in \Gamma(S(T M))$. Since $\alpha \neq \beta$, we have $X \varphi=0$ for all $X \in \Gamma(S(T M))$, that is, $\varphi$ is a constant along $S(T M)$. Take $X, Y \in \Gamma\left(D_{\alpha}^{s}\right)$ in (2.10), we have $\xi[\varphi] \alpha=0$. Also, take $X, Y \in \Gamma\left(D_{\beta}^{s}\right)$ in (2.10), we have $\xi[\varphi] \beta=0$. Since $(\alpha, \beta) \neq(0,0)$, we have $\xi[\varphi]=0$. Thus we have $X \varphi=0$ for all $X \in \Gamma(T M)$, i.e., $\varphi$ is a constant on $M$.

For all $X \in \Gamma\left(D_{\alpha}^{s}\right)$ and $U \in \Gamma\left(D_{\beta}^{s}\right)$, since $\left(\nabla_{X} B\right)(U, Z)=\left(\nabla_{U} B\right)(X, Z)$,

$$
g\left(\left\{\left(A_{\xi}^{*}-\beta P\right) \nabla_{X} U-\left(A_{\xi}^{*}-\alpha P\right) \nabla_{U} X\right\}, Z\right)=0, \forall Z \in \Gamma(S(T M)) .
$$

As $S(T M)$ is non-degenerate, we get $\left(A_{\xi}^{*}-\beta P\right) \nabla_{X} U=\left(A_{\xi}^{*}-\alpha P\right) \nabla_{U} X$. Since the left term of the last equation is in $\Gamma\left(D_{\alpha}^{s}\right)$ and the right term is in $\Gamma\left(D_{\beta}^{s}\right)$ and $D_{\alpha}^{s} \cap D_{\beta}^{s}=\{0\}$, we have $\left(A_{\xi}^{*}-\beta P\right) \nabla_{X} U=0$ and $\left(A_{\xi}^{*}-\alpha P\right) \nabla_{U} X=0$. This imply that $\nabla_{X} U \in \Gamma\left(D_{\beta}\right)$ and $\nabla_{U} X \in \Gamma\left(D_{\alpha}\right)$. On the other hand, $\nabla_{X} U=\nabla_{X}^{*} U$ and $\nabla_{U} X=\nabla_{U}^{*} X$ due to $D_{\alpha} \perp_{B} D_{\beta}$, we have

$$
\nabla_{X} U \in \Gamma\left(D_{\beta}^{s}\right), \quad \nabla_{U} X \in \Gamma\left(D_{\alpha}^{s}\right), \quad \forall X \in \Gamma\left(D_{\alpha}^{s}\right), U \in \Gamma\left(D_{\beta}^{s}\right) .
$$

For $X, Y \in \Gamma\left(D_{\alpha}^{s}\right)$ and $U, V \in \Gamma\left(D_{\beta}^{s}\right)$, since $g(X, U)=0$, we have

$$
g\left(\nabla_{Y} X, U\right)+g\left(X, \nabla_{Y} U\right)=0, \quad g\left(\nabla_{V} U, X\right)+g\left(U, \nabla_{V} X\right)=0 .
$$

Using (3.6), we have $g\left(X, \nabla_{Y} U\right)=g\left(U, \nabla_{V} X\right)=0$. Thus we show that

$$
g\left(\nabla_{Y} X, U\right)=0, \quad g\left(X, \nabla_{V} U\right)=0 .
$$

Since the leaf $M^{*}$ of $S(T M)$ is a semi-Riemannian manifold and $S(T M)=$ $D_{\alpha}^{s} \oplus_{\text {orth }} D_{\beta}^{s}$, where $D_{\alpha}^{s}$ and $D_{\beta}^{s}$ are integrable and parallel distributions with respect to the induced connection $\nabla^{*}$ on $M^{*}$ due to (3.7), by the decomposition theorem of de Rham [13], we have $M^{*}=M_{\alpha} \times M_{\beta}$, where $M_{\alpha}$ and $M_{\beta}$ are some leaves of $D_{\alpha}^{s}$ and $D_{\beta}^{s}$ respectively. Thus we have our theorem.

Theorem 3.2. Let $(M, g, S(T M))$ be a screen conformal half lightlike submanifold of a Lorentzian space form $\left(\bar{M}^{m+3}(c), \bar{g}\right), m>2$, with a conformal Killing coscreen distribution. If $M$ is Einstein, i.e., Ric $=\gamma g$, then $M$ is locally a product manifold $L \times M_{\alpha} \times M_{\beta}$, where $L$ is a null curve and $M_{\alpha}$ and $M_{\beta}$ are totally umbilical leaves of some distributions of $M$ :

(1) If $\gamma \neq(m-1)\left(c+\delta^{2}\right)$, then either $M_{\alpha}$ or $M_{\beta}$ is an m-dimensional Einstein Riemannian space form which is isometric to a sphere $(\gamma>0)$ or a hyperbolic space $(\gamma<0)$ and the other is a point on $M$. 
(2) If $\gamma=(m-1)\left(c+\delta^{2}\right)$, then $M_{\alpha}$ is an $(m-1)$ or m-dimensional Einstein Riemannian space form which is isometric to a sphere $(\gamma>0)$ or a hyperbolic space $(\gamma<0)$ or a Euclidean space $(\gamma=0)$ and $M_{\beta}$ is a spacelike curve or a point on $M$.

Proof. First, we prove that $\gamma=0$ and $\alpha \beta=0$ if $0<p<m$ : If $0<p<m$, then, since $\operatorname{rank} A_{\xi}^{*}>0$, we have $c+\delta^{2}=0$ by Theorem 2.5. If $p=1$ or $p=m-1$, then, from the facts that $(p-1) \alpha+(m-p-1) \beta=0$ and $m>2$, we show that if $p=1$, then $\beta=0$ and if $p=m-1$, then $\alpha=0$. Thus $\gamma=\varphi \alpha \beta=0$. If $1<p<m-1$, then, from (3.7), we know that $\nabla_{U} U$ has no component of $D_{\alpha}$. Since the projection morphism $P$ maps $\Gamma\left(D_{\beta}\right)$ onto $\Gamma\left(D_{\beta}^{s}\right)$ and $S(T M)=D_{\alpha}^{s} \oplus_{\text {orth }} D_{\beta}^{s}$,

$$
\nabla_{U} U=P\left(\nabla_{U} U\right)+\eta\left(\nabla_{U} U\right) \xi, \quad P\left(\nabla_{U} U\right) \in \Gamma\left(D_{\beta}^{s}\right) .
$$

It follows that

$$
\begin{aligned}
g\left(\nabla_{X} \nabla_{U} U, X\right) & =g\left(\nabla_{X} P\left(\nabla_{U} U\right), X\right)+\eta\left(\nabla_{U} U\right) g\left(\nabla_{X} \xi, X\right) \\
& =-\alpha \eta\left(\nabla_{U} U\right) g(X, X) .
\end{aligned}
$$

As $\eta\left(\nabla_{U} U\right)=-\bar{g}\left(U, \bar{\nabla}_{U} N\right)=g\left(U, A_{N} U\right)=\varphi g\left(U, A_{\xi}^{*} U\right)=\varphi \beta g(U, U)$, we get

$$
g(R(X, U) U, X)=-\varphi \alpha \beta g(X, X) g(U, U) .
$$

While, from the Gauss equation (2.3), we have

$$
g(R(X, U) U, X)=\varphi \alpha \beta g(X, X) g(U, U),
$$

due to $c+\delta^{2}=0$. From the last two equations, we get $\gamma=\varphi \alpha \beta=0$.

(1) Let $\gamma \neq(m-1)\left(c+\delta^{2}\right)$ : In this case, we have $c+\delta^{2}=0$. First, in case $s^{2} \neq 4 F$. The equation (3.3) has two non-vanishing distinct solutions $\alpha$ and $\beta$. If $0<p<m$, then $\gamma=0$. This implies that $\gamma=(m-1)\left(c+\delta^{2}\right)$. Therefore, we have $p=0$ or $p=m$. If $p=0$, then $M=L \times M^{*}=L \times\{x\} \times M^{*}$ and $B(X, Y)=g\left(A_{\xi}^{*} X, Y\right)=\beta g(X, Y)$ for any $X, Y \in \Gamma(T M)$. From this and (2.1), we show that $C(X, Y)=\varphi \beta g(X, Y)$ for all $X, Y \in \Gamma(T M)$. Thus $M^{*}$ is totally umbilical. From (2.4) and (2.13), we have

$$
\begin{aligned}
& R^{*}(X, Y) Z=2 \varphi \beta^{2}\{g(Y, Z) X-g(X, Z) Y\}, \\
& \operatorname{Ric}^{*}(X, Y)=2 \varphi \beta^{2}(m-1) g(X, Y), \forall X, Y, Z \in \Gamma(S(T M)) .
\end{aligned}
$$

Thus $M^{*}$ is Einstein and $2 \varphi \beta^{2}$ is a constant due to $m>2$. By $(2.13)$, we have $2 \gamma=2 \varphi \beta^{2}$. Therefore, $M^{*}$ is an Einstein space of constant curvature $2 \gamma$. By duality, if $p=m$, then $M=L \times M^{*}=L \times M^{*} \times\{x\}$ and $B(X, Y)=\alpha g(X, Y)$ for any $X, Y \in \Gamma(T M)$. Thus $M$ is totally umbilical and $M^{*}$ is a totally umbilical Einstein space of constant curvature $2 \gamma=2 \varphi \alpha^{2}$. In case $s^{2}=4 F$. The equation (3.3) has only one non-vanishing solution, named by $\alpha$ and $\alpha$ is a unique eigenvalue of $A_{\xi}^{*}$. In this case, the first equation of (3.4) reduces to $2 \alpha=m \alpha$. This implies $m=2$. Thus this case is an impossible one. 
(2) Let $\gamma=(m-1)\left(c+\delta^{2}\right)$ : The equation (3.3) reduces to $x(x-s)=0$. In case $s \neq 0$. Let $\alpha=0$ and $\beta=s$. Then we have $s=\beta=(m-p) \beta$, i.e., $(m-p-1) \beta=0$. So $p=m-1$. Thus $M_{\alpha}$ is a totally geodesic $(m-1)$ dimensional Riemannian manifold and $M_{\beta}$ is a spacelike curve in $M$. In the sequel, let $X, Y, Z \in \Gamma\left(D_{\alpha}^{s}\right)$ and $U \in \Gamma\left(D_{\beta}^{s}\right)$. From (2.4), we have

$$
\begin{aligned}
& R^{*}(X, Y) Z=\left(c+\delta^{2}\right)\{g(Y, Z) X-g(X, Z) Y\}, \\
& \operatorname{Ric}^{*}(X, Y)=\left(c+\delta^{2}\right)(m-1) g(X, Y) .
\end{aligned}
$$

Thus $g\left(R^{*}(X, Y) Z, U\right)=0$. This implies $\pi_{\alpha} R^{*}(X, Y) Z=R^{*}(X, Y) Z$, where $\pi_{\alpha}$ is the projection morphism of $\Gamma(S(T M))$ on $\Gamma\left(D_{\alpha}^{s}\right)$ and $\pi_{\alpha} R^{*}$ is the curvature tensor of $D_{\alpha}^{s}$. Thus $M_{\alpha}$ is an Einstein manifold of a constant curvature $\left(c+\delta^{2}\right)$. Therefore, $M$ is locally a product $L \times M_{\alpha} \times M_{\beta}$, where $M_{\alpha}$ is an $(m-1)$-dimensional Einstein Riemannian space form of a constant curvature $\left(c+\delta^{2}\right)$ and $M_{\beta}$ is a spacelike curve in $\bar{M}$. In case $s=0$, we get $\alpha=\beta=0$, $A_{\xi}^{*}=B=0$ and $D_{\alpha}^{s}=D_{\beta}^{s}=S(T M)$. Since $M$ is screen conformal, we also have $C=A_{N}=0$. Thus $M^{*}$ is totally geodesic. Using (2.4), we have

$$
R^{*}(X, Y) Z=\left(c+\delta^{2}\right)\{g(Y, Z) X-g(X, Z) Y\}
$$

for all $X, Y, Z \in \Gamma(S(T M))$. Thus $M$ is locally a product $L \times M^{*} \times\{x\}$, where $M^{*}$ is an $m$-dimensional Einstein Riemannian space form of a constant curvature $\left(c+\delta^{2}\right)$ and $\{x\}$ is a point. In these cases, since $\left(c+\delta^{2}\right)=\frac{\gamma}{m-1}$, we have $\operatorname{sgn}\left(c+\delta^{2}\right)=\operatorname{sgn} \gamma$. Thus $M_{\alpha}$ and $M^{*}$ are isometric to spheres (if $\gamma>0$ ) or hyperbolic spaces (if $\gamma<0$ ) or Euclidean spaces (if $\gamma=0$ ).

Corollary 3.3. Let $(M, g, S(T M))$ be a screen conformal Einstein half lightlike submanifold of a Lorentzian space form $\left(\bar{M}^{m+3}(c), \bar{g}\right), m>2$, with a Killing coscreen distribution. Then $M$ is locally a product manifold $L \times M_{\alpha} \times M_{\beta}$, where $L$ is a null curve and $M_{\alpha}$ and $M_{\beta}$ are totally umbilical leaves of some distributions of $M$ :

(1) If $\gamma \neq 0$, either $M_{\alpha}$ or $M_{\beta}$ is an $m$-dimensional Riemannian space form which is isometric to a sphere $(\gamma>0)$ or a hyperbolic space $(\gamma<0)$ and the other is a point in $M$.

(2) If $\gamma=0, M_{\alpha}$ is an $(m-1)$ or $m$-dimensional Euclidean space and $M_{\beta}$ is a spacelike curve or a point in $M$.

Proof. (1) Let $\gamma \neq 0$ : In case $s^{2} \neq 4 F$. If $0<p<m$, then $\gamma=0$. Thus $p=0$ or $p=m$. Either $M_{\alpha}$ or $M_{\beta}$ is a totally umbilical Riemannian manifold $M^{*}$ of constant curvature $2 \varphi \alpha^{2}$ or $2 \varphi \beta^{2}$ respectively due to $\delta=c=0$. Thus $M$ is locally a product manifold $L \times M^{*} \times\{x\}$ or $L \times\{x\} \times M^{*}$, where $M^{*}$ is an $m$-dimensional totally umbilical Riemannian manifold of constant curvature $2 \gamma=2 \varphi \beta^{2}$ or $2 \gamma=2 \varphi \alpha^{2}$ which is isometric to a sphere or a hyperbolic space according to the sign of $\gamma$ and $\{x\}$ is a point. The case $s^{2}=4 F$ is not appear because $m>2$. 
(2) Let $\gamma=0$ : In case $s \neq 0$. Then $\alpha=0$ and $\beta=s$. Since $p=m-1, M_{\alpha}$ is an $(m-1)$-dimensional Riemannian manifold of curvature $c+\delta^{2}=0$ and $M_{\beta}$ is a spacelike curve. Thus $M$ is locally a product manifold $L \times M_{\alpha} \times M_{\beta}$, where $M_{\alpha}$ is an $(m-1)$-dimensional Euclidean space and $M_{\beta}$ is a spacelike curve in $M$. In case $s=0$. Then $\alpha=\beta=0$ and $D_{\alpha}^{s}=D_{\beta}^{s}=S(T M)$. Thus $M^{*}$ is an $m$-dimensional Riemannian manifold of curvature $c+\delta^{2}=0$. Thus $M$ is locally a product $L \times M^{*} \times\{x\}$ where $M^{*}$ is an $m$-dimensional Euclidean space, $L$ is a null curve and $\{x\}$ is a point.

Example 3. Consider a surface $M$ in $R_{2}^{4}$ given by the equations

$$
x_{3}=\frac{1}{\sqrt{2}}\left(x_{1}+x_{2}\right), \quad x_{4}=\frac{1}{2} \ln \left(1+\left(x_{1}-x_{2}\right)^{2}\right) .
$$

Then $T M=\operatorname{Span}\{U, V\}$ and $T M^{\perp}=\operatorname{Span}\{\xi, u\}$, where we set

$$
\begin{aligned}
& U=\sqrt{2}\left(1+\left(x_{1}-x_{2}\right)^{2}\right) \partial_{1}+\left(1+\left(x_{1}-x_{2}\right)^{2}\right) \partial_{3}+\sqrt{2}\left(x_{1}-x_{2}\right) \partial_{4}, \\
& V=\sqrt{2}\left(1+\left(x_{1}-x_{2}\right)^{2}\right) \partial_{2}+\left(1+\left(x_{1}-x_{2}\right)^{2}\right) \partial_{3}-\sqrt{2}\left(x_{1}-x_{2}\right) \partial_{4}, \\
& \xi=\partial_{1}+\partial_{2}+\sqrt{2} \partial_{3}, \\
& u=2\left(x_{2}-x_{1}\right) \partial_{2}+\sqrt{2}\left(x_{2}-x_{1}\right) \partial_{3}+\left(1+\left(x_{1}-x_{2}\right)\right) \partial_{4} .
\end{aligned}
$$

By direct calculations we check that $\operatorname{Rad}(T M)$ is a distribution on $M$ of rank 1 spanned by $\xi$. Hence $M$ is a half-lightlike submanifold of $R_{2}^{4}$. Choose $S(T M)$ and $S\left(T M^{\perp}\right)$ spanned by $V$ and $u$ which are timelike and spacelike respectively. We obtain the lightlike transversal vector bundle

$$
\operatorname{ltr}(T M)=\operatorname{Span}\left\{N=-\frac{1}{2} \partial_{1}+\frac{1}{2} \partial_{2}+\frac{1}{\sqrt{2}} \partial_{3}\right\},
$$

and the transversal bundle $\operatorname{tr}(T M)=\operatorname{Span}\{N, u\}$. Denote by $\bar{\nabla}$ the LeviCivita connection on $R_{2}^{4}$ and by straightforward calculations we obtain

$$
\begin{aligned}
& \bar{\nabla}_{V} V=2\left(1+\left(x_{1}-x_{2}\right)^{2}\right)\left\{2\left(x_{2}-x_{1}\right) \partial_{2}+\sqrt{2}\left(x_{2}-x_{1}\right) \partial_{3}+\partial_{4}\right\}, \\
& \bar{\nabla}_{\xi} V=0, \quad \bar{\nabla}_{X} \xi=\bar{\nabla}_{X} N=0, \quad \forall X \in \Gamma(T M) .
\end{aligned}
$$

Taking into account of Gauss and Weingarten formulae, we infer

$$
\begin{aligned}
& B=0, \quad A_{\xi}^{*}=0, \quad A_{N}=0, \quad \nabla_{X} \xi=0, \quad \tau(X)=\rho(X)=0, \\
& {\left[D(X, \xi)=0, \quad D(V, V)=2, \quad \nabla_{X} V=\frac{2 \sqrt{2}\left(x_{2}-x_{1}\right)^{3}}{1+\left(x_{1}-x_{2}\right)^{2}} X^{2} V\right.}
\end{aligned}
$$

for any $X=X^{1} \xi+X^{2} V$ tangent to $M$. As $A_{\xi}^{*} X=A_{N} X=0$ for any $X \in$ $\Gamma(T M), M$ is a trivial screen conformal half lightlike submanifold of $R_{2}^{4}$. Since $g(V, V)=-\left(1+\left(x_{1}-x_{2}\right)^{4}\right)$ we have

$$
D(V, V)=\delta g(V, V), \quad \text { where } \quad \delta=-\frac{2}{\left(1+\left(x_{1}-x_{2}\right)^{4}\right)} .
$$


Therefore $M$ is a screen conformal half lightlike submanifold of $R_{2}^{4}$ with a conformal Killing coscreen distribution $S\left(T M^{\perp}\right)$. Thus $M$ is locally a product manifold $M=L_{1} \times L_{2}$, where $L_{1}$ is a null curve tangent to $\operatorname{Rad}(T M)$ and $L_{2}$ is a timelike curve tangent to $S(T M)$.

\section{References}

[1] C. Atindogbe and K. L. Duggal, Conformal screen on lightlike hypersurfaces, Int. J. Pure Appl. Math. 11 (2004), no. 4, 421-442.

[2] J. K. Beem, P. E. Ehrlich, and K. L. Easley, Global Lorentzian Geometry, Marcel Dekker, Inc. New York, Second Edition, 1996.

[3] B. Y. Chen, Geometry of Submanifolds, Marcel Dekker, New York, 1973.

[4] K. L. Duggal and A. Bejancu, Lightlike submanifolds of codimension two, Math. J. Toyama Univ. 15 (1992), 59-82.

[5] — Lightlike Submanifolds of Semi-Riemannian Manifolds and Applications, Kluwer Acad. Publishers, Dordrecht, 1996.

[6] K. L. Duggal and D. H. Jin, Half-lightlike submanifolds of codimension two, Math. J. Toyama Univ. 22 (1999), 121-161.

[7] A. Fialkow, Hypersurfaces of a space of constant curvature, Ann. of Math. (2) 39 (1938), no. $4,762-785$.

[8] S. G. Harris, A triangle comparison theorem for Lorentz manifolds, Indiana Univ. Math. J. 31 (1982), no. 3, 289-308.

[9] D. H. Jin, Einstein half lightlike submanifolds with a Killing co-screen distribution, Honam Math. J. 30 (2008), no. 3, 487-504.

[10] — A characterization of screen conformal half lightlike submanifolds, Honam Math. J. 31 (2009), no. 1, 17-23.

[11] D. N. Kupeli, Singular Semi-Riemannian Geometry, Mathematics and Its Applications, Kluwer Acad. Publishers, Dordrecht, 1996.

[12] B. O'Neill, Semi-Riemannian Geometry with Applications to Relativity, Academic Press, 1983.

[13] G. de Rham, Sur la réductibilité d'un espace de Riemannian, Comment. Math. Helv. 26 (1952), 328-344.

[14] T. Y. Thomas, On closed spaces of constant mean curvature, Amer. J. Math. 58 (1936), no. $4,702-704$.

[15] K. Yano, Differential Geometry on Complex and Almost Complex Spaces, The Macmillan Company, 1965.

Department of Mathematics

DONGGUK UNIVERSITY

KYONGJu 780-714, KoreA

E-mail address: jindh@dongguk.ac.kr 